\title{
Spatially resolved submillimeter imaging of the HR 8799 debris disk ${ }^{\star, \star \star}$
}

\author{
J. Patience ${ }^{1}$, J. Bulger ${ }^{1}$, R. R. King ${ }^{1}$, B. Ayliffe ${ }^{1}$, M. R. Bate ${ }^{1}$, I. Song ${ }^{2}$,

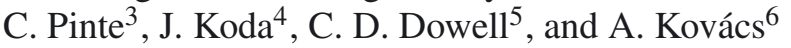

\author{
${ }^{1}$ Astrophysics Group, College of Engineering, Mathematics and Physical Sciences, University of Exeter, Exeter, EX4 4QL, UK \\ e-mail: joanna@astro.ex.ac.uk \\ 2 Department of Physics and Astronomy, University of Georgia, Athens, GA 30602-2451, USA \\ 3 UJF-Grenoble 1/CNRS-INSU, Institut de Plantologie et d'Astrophysique de Grenoble, UMR 5274, Grenoble, 38041, France \\ ${ }^{4}$ Department of Physics and Astronomy, Stony Brook University, Stony Brook, NY 11794-3800, USA \\ 5 Department of Physics and Astronomy, Caltech, MC 249-17, Pasadena, CA 91125, USA \\ ${ }^{6}$ School of Physics \& Astronomy, Tate Lab Room 148, 116 Church Street S.E., Minneapolis, MN 55455, USA
}

Received 1 June 2011 / Accepted 21 June 2011

\section{ABSTRACT}

\begin{abstract}
Dynamical interactions between planets and debris disks may sculpt the disk structure and impact planetary orbits, but only a few systems with both imaged planets and spatially resolved debris disks are known. With the Caltech Submm Observatory (CSO), we have observed the HR 8799 debris disk at $350 \mu \mathrm{m}$. The $350 \mu \mathrm{m}$ map is the first spatially resolved measurement of the debris disk encircling the HR 8799 planetary system at this wavelength. Both the flux and size of the emission are consistent with a Kuiper belt of dust extending from $\sim 100-300 \mathrm{AU}$. Although the resolution of the current map is limited, the map shows an indication of offset asymmetric emission, and several scenarios for this possibility are explored with radiative transfer calculations of a star-disk system and $N$-body numerical simulations of planet-disk interactions with parameters representative of the HR 8799 system.
\end{abstract}

Key words. planetary systems - planet-disk interactions - submillimeter: planetary systems - radiative transfer

\section{Introduction}

Planets and debris disks are intimately linked, as planets can scatter or trap planetesimals in a disk (Mouillet et al. 1997), and planetestimals may drive planet migration (Kirsh et al. 2009). Three planetary systems have been imaged orbiting dusty A-stars (Marois et al. 2008; Kalas et al. 2008; Lagrange et al. 2010) with remnant debris disks (Smith \& Terrile 1984; Holland et al. 1998; Zuckerman \& Song 2004) sustained by the collisional grinding of planetesimals into smaller particles (Backman $\&$ Paresce 1993). These systems present rare cases in which it is possible to explore the dynamical effects on disk structure of planets with known locations. Structures such as asymmetries and clumps can encode the effects of gravitational interactions (e.g. Liou \& Zook 1999; Kuchner \& Holman 2003; Wyatt 2006; Quillen \& Faber 2006). The submm/mm wavelength range is ideal for studying the interaction of the disk and planets, since the large grains are expected to remain in resonances with the planets, while the smaller grains evolve into axisymmetric structures due to scattering or radiation pressure (Wyatt 2006).

From IRAS photometry, HR 8799 was identified as a debris disk system with excess emission above the stellar photosphere at $60 \mu \mathrm{m}$, consistent with cool dust (Zuckerman \& Song 2004). With sensitive Spitzer measurements over the $5.5 \mu \mathrm{m}-35 \mu \mathrm{m}$ range, the disk was further estimated to contain two components: a hotter inner ring of dust analogous to the asteroid belt with an inner radius of $6 \mathrm{AU}$ and a cooler outer disk (Chen et al. 2006).

\footnotetext{
* Based on observations obtained at the Caltech Submillimeter Observatory.

$\star \star$ Figures 3 and 4 are available in electronic form at http://www . aanda.org
}

The presence of dust, combined with a young age estimated from the colour-magnitude diagram, made HR 8799 a target for highcontrast imaging which revealed a system of four giant planets at projected separations ranging from 14-70 AU (Marois et al. $2008,2010)$. The planets are located between the two dust populations. Subsequent Spitzer images at $70 \mu \mathrm{m}$ resolved a distribution of dust at very large radii from HR 8799, and this emission may probe smaller blown out grains (Su et al. 2009). This letter reports the first observations of the disk at $350 \mu \mathrm{m}$, including a spatially resolved map and a comparison with numerical models.

\section{Observations}

Submillimeter observations of HR 8799 were acquired with the $10 \mathrm{~m}$ Caltech Submillimeter Observatory (CSO) with the SHARCII instrument (Dowell et al. 2003) which includes a $12 \times 32$ bolometer array with a $>90 \%$ filling factor. At the observation wavelength of $350 \mu \mathrm{m}$, the beam size is $\sim 8^{\prime \prime} .5$ and the shape is stabilised by the Dish Surface Optimization System (DSOS) which corrects for dish figure changes as a function of telescope elevation. The Lissajous scanning pattern employed for the observations resulted in a map size of $\sim 3 ! 3 \times 2 !$. The data were acquired over two nights with excellent tau conditions $\left(\tau_{225 \mathrm{GHz}}=0.03-0.04\right)$ on 6 and 7 Aug. 2007. Follow-up telescope time allocated to obtain deeper maps did not have sufficient tau conditions. For the observing sequence, a series of 4-6 scans of $300 \mathrm{~s}$ to $620 \mathrm{~s}$ each were obtained on the target and were preceded and followed by $120 \mathrm{~s}$ scans of a bright pointing and flux calibrator, Uranus, Neptune, or the quasar 3C 454.3. In total, 20 scans were taken on the science target for a total of $2.75 \mathrm{~h}$ of data. Given the importance of calibration, a similar 
number of 23 scans of calibrators were recorded over the time period of the observations. The target and calibrators sampled a similar range of temperature, elevation, and opacity.

\section{Data analysis}

The construction of the CSO map required two main steps - calculating pointing offsets and extracting the signal from the background. For each scan, a pointing correction was first calculated by first computing and then applying pointing corrections that account for both static and time variable pointing drifts, using calibrator scans, a pointing model ${ }^{1}$ and software. The calibration scans were used in conjunction with the pointing model to calculate the pointing offset applicable at the time and sky position of each target observation. The pointing corrections, along with the measurements of the atmospheric opacity at the time of observation, were then included in the data processing using the software CRUSH, version 2.01-4 (Kovács 2008). In the final map, the total flux was measured within the $3 \sigma$ contour using the MIRIAD (Sault et al. 1995) software. The absolute flux calibration was based on the scans of the planet Uranus and the planet flux at the observation date from the JPL Horizons model. The total uncertainty in the HR 8799 flux is $35 \%$, a combination of the small $(6 \%)$ variation in the Uranus flux measurements and the rms noise level in measured in the final source map.

To quantify the location of the emission peak, the distance from the host star position to the brightest part of the disk was measured. Since the photosphere of HR 8799 is not detectable at $350 \mu \mathrm{m}$, it was necessary to identify the location of the central source based on the telescope pointing. The bright calibration targets were used to empirically measure the pointing accuracy. For the pointing test, each calibrator scan was reduced in a manner analogous to the targets - i.e. by removing that calibrator scan (and others taken within a few minutes) from the pointing correction calculation and deriving a new pointing correction from the remaining calibrators and pointing model and then applying the pointing offset to the CRUSH reduction. This approach should be conservative in calculating the pointing offset to apply to the calibrator scan since the calibrators for the target are taken at most $30 \mathrm{~min}$ before/after the targets, while this approach for the calibrators has mainly included calibrators $\sim 60$ min before/after each calibrator scan treated as a pointing test. The absolute value of the pointing error for each calibrator analysed with this procedure was measured from the difference of the position of the calibrator in each map to its known coordinates. Since we are interested in the central position of the source, we calculated the standard deviation of the mean of the absolute offsets. Based on this empirical test, the position uncertainty is \pm 0 .' 6 . Since the position of the emission peak is identified within a fraction of a pixel in the final map, the source position uncertainty dominates the uncertainty on distance measurements.

\section{Numerical modelling}

To interpret the debris disk map, the flux and morphology were compared with three numerical models - a radiative transfer model of a star surrounded by symmetric zones of dust, a simulation of massive planets migrating outwards and interacting with planetesimals and dust, and a simulation from the literature of a low mass planet interacting with planetesimals. The Monte Carlo $3 \mathrm{D}$ continuum radiative transfer code MCFOST (Pinte et al. 2006) was used to generate SED models and images at $350 \mu \mathrm{m}$.

\footnotetext{
1 www. submm.caltech.edu/sharc/analysis/pmodel/
}

In the MCFOST routines, the photons from the central star with properties given in Reidemeister et al. (2009) were propagated through the disk with a model incorporating a combination of Mie theory scattering, absorption, and re-emission. The disk parameters used to construct the SED model are given in Table 1. The disk zones are based on the 3-component model described in $\mathrm{Su}$ et al. (2009). Minor modifications were included to account for the dynamically cleared chaotic zones around the innermost and outermost planet (Quillen \& Faber 2006; Moro-Martín et al. 2010; Fabrycky \& Murray-Clay 2010). A series of simulated images were generated for a range of outer disk radii and inclinations, and Table 1 shows the values most consistent with the CSO map.

The structure in the CSO map was also compared with numerical models performed using an $N$-body code. Our numerical model included the interaction of planets resembling HR 8799b (6 $M_{\text {Jup }}$ ) (Currie et al. 2011) and HR 8799c (8 $M_{\text {Jup }}$ ) (Currie et al. 2011) migrating outwards at the same rate through a disk of planetesimals. Since there was no significant difference in the results when two planets were included rather than one, the planets more distant planets from the debris disk should have no impact and were not added to the simulation. The initial planetesimal belt had a width of $5 \mathrm{AU}$ and an inner radius of $91 \mathrm{AU}$, and the planets migrated $15 \mathrm{AU}$ to their final orbits. Once the two planets had reached approximately their present day orbits (Marois et al. 2008), the orbits were circularised, and the planetesimal disk replaced by a dust disk. The stability of the planetesimals in a very similar configuration has already been shown in previous simulations (Moro-Martín et al. 2010).

The transformation of planetesimals to dust entailed the introduction of radiation pressure and Poynting-Robertson drag through the parameter $\beta$ that quantifies the ratio of radiation to gravitational forces (Burns et al. 1979). The grains responsible for the $350 \mu \mathrm{m}$ emission have a size distribution peaked near $350 \mu \mathrm{m}$, so the $\beta$ value appropriate for the map is 0.0055 . The model is designed to investigate the inner region of the Kuiper belt and does not include the entire disk structure or all three components of the SED model. Simulated surface density maps of the inner Kuiper belt were produced at a range of wavelengths. Finally, the CSO map structure was also considered in the context of results of dynamical simulations of a low mass planet interacting with a planetesimal belt (Reche et al. 2008)

\section{Results and discussion}

\subsection{Disk flux and size}

The CSO map is given in Fig. 1, and the measured flux for the disk integrated over the $3 \sigma$ contour is $89 \pm 26 \mathrm{mJy}$. The $350 \mu \mathrm{m}$ map of the HR 8799 debris disk reveals emission that is extended compared to the maps of the bright point source calibrators taken on the same night. Only spatially resolved images of dust with small $\beta$ values are capable of distinguishing the location of the dust based on the structure, since the SED solution for the disk radius is degenerate with dust properties such as $a_{\text {min }}$. The inner disk is not resolved, but set at $100 \mathrm{AU}$, based on previous simulations of orbital stability (Moro-Martín et al. 2010) and the mass of the outermost planet.

To estimate the size of the dust belt, a series of MCFOST models were run with a range of outer disk radii. All models simultaneously matched the $350 \mu \mathrm{m}$ flux and previously measured fluxes reported in Su et al. (2009) or compiled by Reidemeister et al. (2009), plotted in Fig. 2. Although the map resolution and sensitivity are limited, the disk outer radius is consistent with 

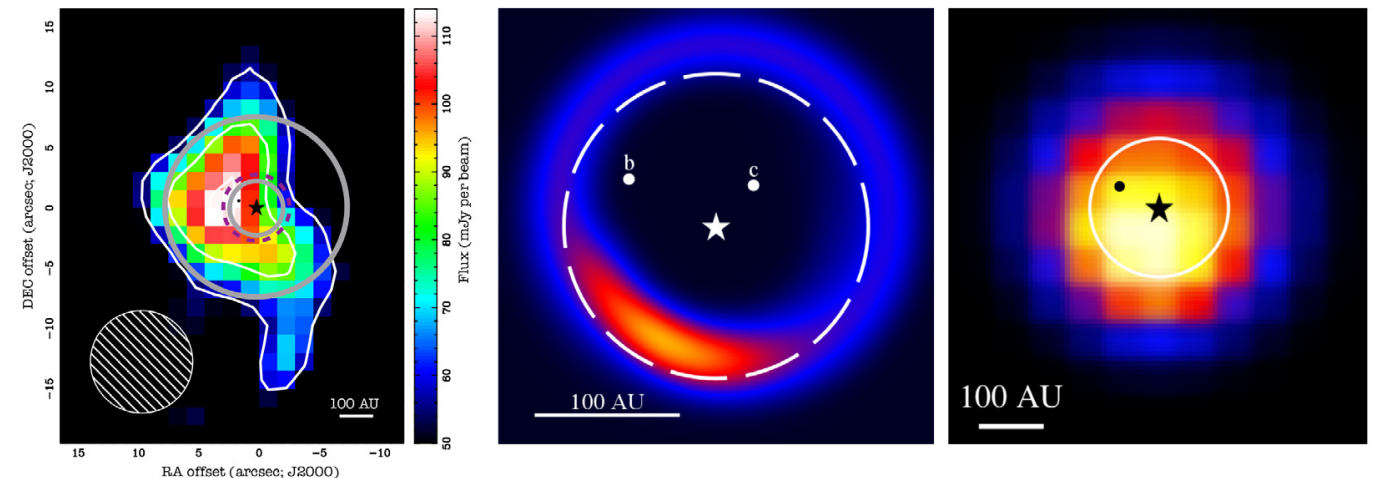

Fig. 1. (left) The CSO $350 \mu \mathrm{m}$ image with the positions of the star (black star) and outermost planet (dot) indicated. The solid circles trace the locations of the inner and outer edge of the Kuiper belt used in the SED model and the dashed line shows the expected position of the 2:1 resonance. (middle) The disk surface density map from the simulation. The semi-major axis centres around the 2:1 resonance with the outer planet (dashed line), and the moderate eccentricities $(\sim 0.3)$ of the dust grains explain their displacement from the circular path. (right) The same numerical simulation convolved with an 8.'5 Gaussian to compare with the brightness peak position in the CSO map of the same resolution. The 2:1 resonance is indicated by the white line.

Table 1. MCFOST disk model parameters.

\begin{tabular}{lccc}
\hline \hline $\begin{array}{l}\text { Model } \\
\text { parameter }^{a}\end{array}$ & $\begin{array}{c}\text { Asteroid } \\
\text { belt }\end{array}$ & $\begin{array}{c}\text { Kuiper } \\
\text { belt }\end{array}$ & $\begin{array}{c}\text { Outer } \\
\text { halo }\end{array}$ \\
\hline$M_{\text {dust }}\left[M_{\odot}\right]$ & $3.3 \times 10^{-12}$ & $3.6 \times 10^{-7}$ & $5.7 \times 10^{-8}$ \\
$r_{\text {in }}[\mathrm{AU}]$ & 6 & 100 & 300 \\
$r_{\text {out }}[\mathrm{AU}]$ & 8 & 300 & 1000 \\
$H_{\mathrm{o}}\left(r_{\text {in }}\right)[\mathrm{AU}]$ & 0.6 & 9 & 30 \\
$H(r)$ & $\sim r^{0}$ & $\sim r^{0}$ & $\sim r^{0}$ \\
$\Sigma(r)$ & $\sim r^{0}$ & $\sim r^{0}$ & $\sim r^{-1}$ \\
$a_{\min }[\mu \mathrm{m}]$ & 2.0 & 3.0 & 1.0 \\
$a_{\max }[\mu \mathrm{m}]$ & 4.5 & 1000 & 10 \\
$\mathrm{~d} n / \mathrm{d} a$ & $\sim a^{-3.5}$ & $\sim a^{-3.5}$ & $\sim a^{-3.5}$ \\
\hline
\end{tabular}

Notes. ${ }^{(a)}$ dust mass $M_{\text {dust }}$, inner radius $r_{\text {in }}$, outer radius $r_{\text {out }}$, scale height $H_{0}$ at a reference radius $\mathrm{r}_{o}$, flaring profile exponent for the disk height $H(r)$, surface density profile $\Sigma(r)$, minimum grain size $a_{\min }$, maximum grain size $a_{\max }$ and the differential grain size distribution $\mathrm{d} n / \mathrm{d} a$.

$\sim 300 \mathrm{AU}$, and this radius is marked on Fig. 1, along with the assumed inner radius. The $350 \mu \mathrm{m}$ emission is largely confined within a radius of $300 \mathrm{AU}$. Further details on the set of simulated $350 \mu \mathrm{m}$ images from MCFOST are given in Fig. 3. A radius of $200 \mathrm{AU}$ results in a more compact structure inconsistent with the data, though higher sensitivity maps will be required to rule out disks much larger than $300 \mathrm{AU}$, since the surface brightness of the most extended emission is beyond the dynamic range of the CSO data.

\subsection{Disk structure}

The observed disk morphology is compared with two types of models of the disk structure - a symmetric dust distribution simulated with MCFOST and an asymmetric dust distribution governed by gravitational interactions and radiation pressure. Assuming a symmetric circular ring for the disk described in Table 1, the effect of changing the inclination is explored in Fig. 3. As the emission is optically thin at $350 \mu \mathrm{m}$ (unlike scattered light images at shorter wavelengths), the disk pattern remains symmetric as the inclination increases. Since the observed map is not well matched by these symmetric patterns, it is not possible to place a strong constraint on the disk inclination to compare with estimates from planet astrometry (Lafrenière et al. 2009), $70 \mu \mathrm{m}$ emission ( $\mathrm{Su}$ et al. 2009), and stability (Moro-Martín et al. 2010).

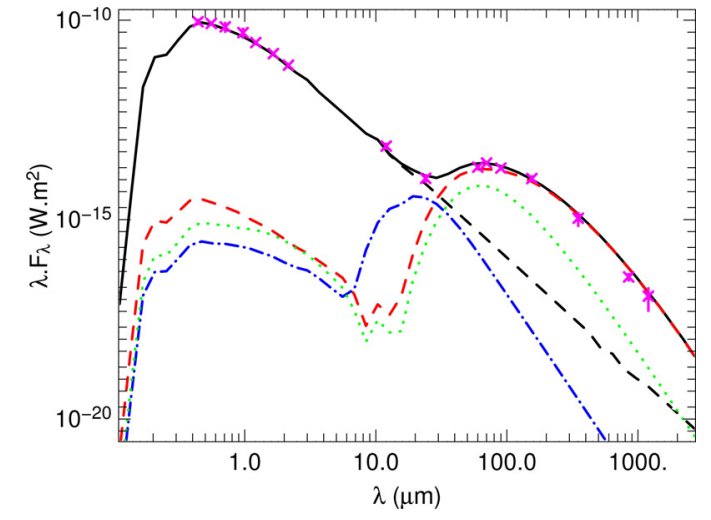

Fig. 2. Fluxes of the HR 8799 star/disk system are plotted as a function of wavelength (purple crosses), along with the SED model with the parameters listed in Table 1 (solid black line). Contributions from the stellar flux (black dashed line) and scattered and emitted light from the asteroid belt (blue dash-dot line), the Kuiper belt (dashed red line), and outer halo (green dotted line).

The CSO map is also compared with numerical models resulting in an asymmetric dust distribution. In addition to the extended structure, the HR 8799 disk emission at $350 \mu \mathrm{m}$ is slightly offset from the coordinates of the host star. The distance from the star to the brightest point in the disk is $2{ }^{\prime \prime} 1 \pm 0.7$, or $84 \pm$ 28 AU. While the uncertainty is too large to assign a specific value to the radius of the brightest region of the disk, an estimate can be compared with the distance to the peak in surface density of the numerical simulation in Fig. 1. A dominant one-sided arc of higher surface density describes the distribution of grains with $\beta$ values on the order of 0.005 or less, as they become trapped in the 2:1 mean motion resonance (Wyatt 2006), based on previously published simulations of migrating planets with masses up to $1 M_{\text {Jup }}$ (Wyatt 2006). Our simulation of a planetesimal belt is a test case extending to include two higher mass planets; a similar asymmetric pattern is evident in our dust surface density map in Fig. 1. The CSO map of the debris disk shows a structure suggestive of a single bright clump like the pattern produced by dust grains trapped in the $2: 1$ mean motion resonance with a planet, while the map does not reveal two distinct clumps separated by 180 degrees, as expected for material trapped in the 3:2 resonance (Wyatt 2006). Within the uncertainty of the measurement, the radius of the brightest region of the disk is consistent with the peak in the surface density image in our simulation; 
a convolution of the simulated map with the CSO beam showing a similar offset is given in Fig. 1.

Based on previous simulations, resonant trapping cannot easily explain that the brightest clump leads rather than lags the planet position, since the libration point lagging position has a higher probability to be filled for an outward migration with a planet with eccentricity greater than 0.03 (Wyatt 2006). In our simulation of a planetesimal belt surrounding two massive planets with slow outward migration, the azimuthal angle of the bulge is subject to large scale oscillations over the course of several orbits, moving between a leading and a trailing position in a continuous manner, without crossing in front of the planet. Such an oscillating structure can be produced if the orbits of the planets become eccentric as they migrate (due to mutual interactions) and, once formed, the structure can persist even if the eccentricities of the planetary orbits are later damped.

If dynamical interactions are governing the particle spatial distribution such that the $350 \mu \mathrm{m}$-emitting grains are trapped in a 2:1 mean motion resonance with the outermost planet, then there are important implications for the orbital history of HR 8799b. To enhance the population of planetesimals in the resonance, orbital migration is required, although the rate of migration cannot be too high, or the trapping probability will decrease (Reche et al. 2008). Planet migration may have strongly influenced the timing and mass flux of the Late Heavy Bombardment of the terrestrial planets in the Solar System (Gomes et al. 2005), so evidence of orbital migration may be an important factor for the conditions in the as-yet unexplored terrestrial planet region in the HR 8799 system. Theoretical arguments favouring Currie et al. (2011) and rejecting Dodson-Robinson et al. (2009) orbital migration for the HR 8799 system have also been proposed. For a resonant pattern to persist, the eccentricity of the orbit of the planet needs to be low, since the libration amplitude of the planetesimals in resonance is increased as eccentricity increases, causing the distribution to become smooth (Reche et al. 2008). Numerical simulations of the HR 8799 planet system (prior to the discovery of the innermost planet, Marois et al. 2010), have identified a set of possible stable orbital solutions, including two with low eccentricities for HR $8799 \mathrm{~b}$ of 0.008 or 0.014 (Goździewski \& Migaszewski 2009). The clumped structure of the CSO map favours these low eccentricity solutions. For comparison, the eccentricity of the orbit of Neptune is 0.0112 and the migration of Neptune may have caused the objects to move into the resonances (Malhotra 1993).

An alternate, non-resonant mechanism for producing a single disk clump is the presence of an outer, low mass planet on an eccentric orbit (Reche et al. 2008), an interesting possibility for a system that already includes four giant planets. In this case, the planet mass would be very low, comparable to an Earth mass, the eccentricity would be large $(\sim 0.4-0.7)$ and the clump would appear at apastron. The lifetime for non-resonant structures is expected to be lower, on the order of $35 \mathrm{Myr}$ (Reche et al. 2008), though the recent assessment of the HR 8799 age of $30 \mathrm{Myr}$ (Zuckerman et al. 2011) is within the feature lifetime, and the system is old enough to have passed from the runaway growth to the oligarchic growth phase of terrestrial planet formation, with enough time for terrestrial planet formation (Chambers 2001).

\section{Future prospects}

The CSO map at $350 \mu \mathrm{m}$ has shown possible indications of dynamical sculpting of the HR 8799 debris disk from the gravitational influence of the outermost planet, and future multi-wavelength observations should reveal even finer details. Simulations of the surface density distribution of dust particles emitting at $24 \mu \mathrm{m}, 70 \mu \mathrm{m}, 350 \mu \mathrm{m}$, and $850 \mu \mathrm{m}$ are given in Fig. 4. At the shortest wavelength of $24 \mu \mathrm{m}$, a symmetric ring is expected and may be resolvable with $8 \mathrm{~m}$-class telescopes, though below the limit of Spitzer. Both Herschel operating at $70 \mu \mathrm{m}$ and SCUBA2 at $450 \mu \mathrm{m}$ have resolution limits comparable to the CSO, and the $70 \mu \mathrm{m}$ emission may be more distributed than the $350 \mu \mathrm{m}$ map. The ALMA and SMA interferometers operate at $450 \mu \mathrm{m}$ and $850 \mu \mathrm{m}$ with higher positional accuracy than a single dish telescope, and the $850 \mu \mathrm{m}$ peak may be offset from the $450 \mu \mathrm{m}$ peak location. ALMA, in particular, will have significantly higher sensitivity to improve the measurement of the disk structure, and will be ideal to pursue deeper, high fidelity maps for further insights into the planet formation process in this benchmark system.

Acknowledgements. We gratefully acknowledge support to Exeter from the Leverhulme Trust (F/00144/BJ ) and STFC (ST/F0071241/1, ST/H002707/1) and to IPAG from EC's 7th Framework Program (PERG06-GA-2009-256513) and ANR of France (ANR-2010-JCJC-0504-01). We acknowledge collaborative support through the EC CONSTELLATION Network and helpful conversation with H. Beust and J.-C. Augereau. Observations at the Caltech Submillimeter Observatory have been supported by NSF grant AST 05-40882 and 08-38261. We thank the referee for helpful comments.

\section{References}

Backman, D. E., \& Paresce, F. 1993, In: Protostars and planets III (A93-42937 17-90), 1253

Burns, J. A., Lamy, P. L., \& Soter, S. 1979, Icarus, 40, 1

Chambers, J. E. 2001, Icarus, 152, 205

Chen, C. H., Sargent, B. A., Bohac, C., et al. 2006, ApJS, 166, 351

Currie, T., Burrows, A., Itoh, Y., et al. 2011, ApJ, 729, 128

Dodson-Robinson, S. E., Veras, D., Ford, E. B., \& Beichman, C. A. 2009, ApJ, 707,79

Dowell, C. D., Allen, C. A., Babu, R. S., et al. 2003, Millimeter and Submillimeter Detectors for Astronomy, ed. Phillips, 4855, 73

Fabrycky, D. C., \& Murray-Clay, R. A. 2010, ApJ, 710, 1408

Gomes, R., Levison, H. F., Tsiganis, K., \& Morbidelli, A. 2005, Nature, 435, 466 Goździewski, K., \& Migaszewski, C. 2009, MNRAS, 397, L16

Holland, W. S., Greaves, J. S., Zuckerman, B., et al. 1998, Nature, 392, 788

Kalas, P., Graham, J. R., Chiang, E., et al. 2008, Science, 322, 1345

Kirsh, D. R., Duncan, M., Brasser, R., \& Levison, H. F. 2009, Icarus, 199, 197

Kovács, A. 2008, Millimeter and Submillimeter Detectors and Instrumentation for Astronomy IV, ed. Duncan, 7020, 45

Kuchner, M. J., \& Holman, M. J. 2003, ApJ, 588, 1110

Lafrenière, D., Marois, C., Doyon, R., \& Barman, T. 2009, ApJ, 694, L148 Lagrange, A.-M., Bonnefoy, M., Chauvin, G., et al. 2010, Science, 329, 57 Liou, J.-C., \& Zook, H. A. 1999, AJ, 118, 580

Malhotra, R. 1993, Nature, 365, 819

Marois, C., Macintosh, B., Barman, T., et al. 2008, Science, 322, 1348

Marois, C., Zuckerman, B., Konopacky, Q. M., Macintosh, B., \& Barman, T. 2010, Nature, 468, 1080

Moro-Martín, A., Rieke, G. H., \& Su, K. Y. L. 2010, ApJ, 721, L199

Mouillet, D., Larwood, J. D., Papaloizou, J. C. B., \& Lagrange, A. M. 1997, MNRAS, 292, 896

Pinte, C., Ménard, F., Duchêne, G., \& Bastien, P. 2006, A\&A, 459, 797

Quillen, A. C., \& Faber, P. 2006, MNRAS, 373, 1245

Reche, R., Beust, H., Augereau, J.-C., \& Absil, O. 2008, A\&A, 480, 551

Reidemeister, M., Krivov, A. V., Schmidt, T. O. B., et al. 2009, A\&A, 503, 247

Sault, R. J., Teuben, P. J., \& Wright, M. C. H. 1995, Astronomical Data Analysis Software and Systems IV, 77, 433

Smith, B. A., \& Terrile, R. J. 1984, Science, 226, 1421

Su, K. Y. L., Rieke, G. H., Stapelfeldt, K. R., et al. 2009, ApJ, 705, 314

Wyatt, M. C. 2006, ApJ, 639, 1153

Zuckerman, B., \& Song, I. 2004, ApJ, 603, 738

Zuckerman, B., Rhee, J. H., Song, I., \& Bessell, M. S. 2011, ApJ, 732, 61

Page 5 is available in the electronic edition of the journal at http://www . aanda.org 

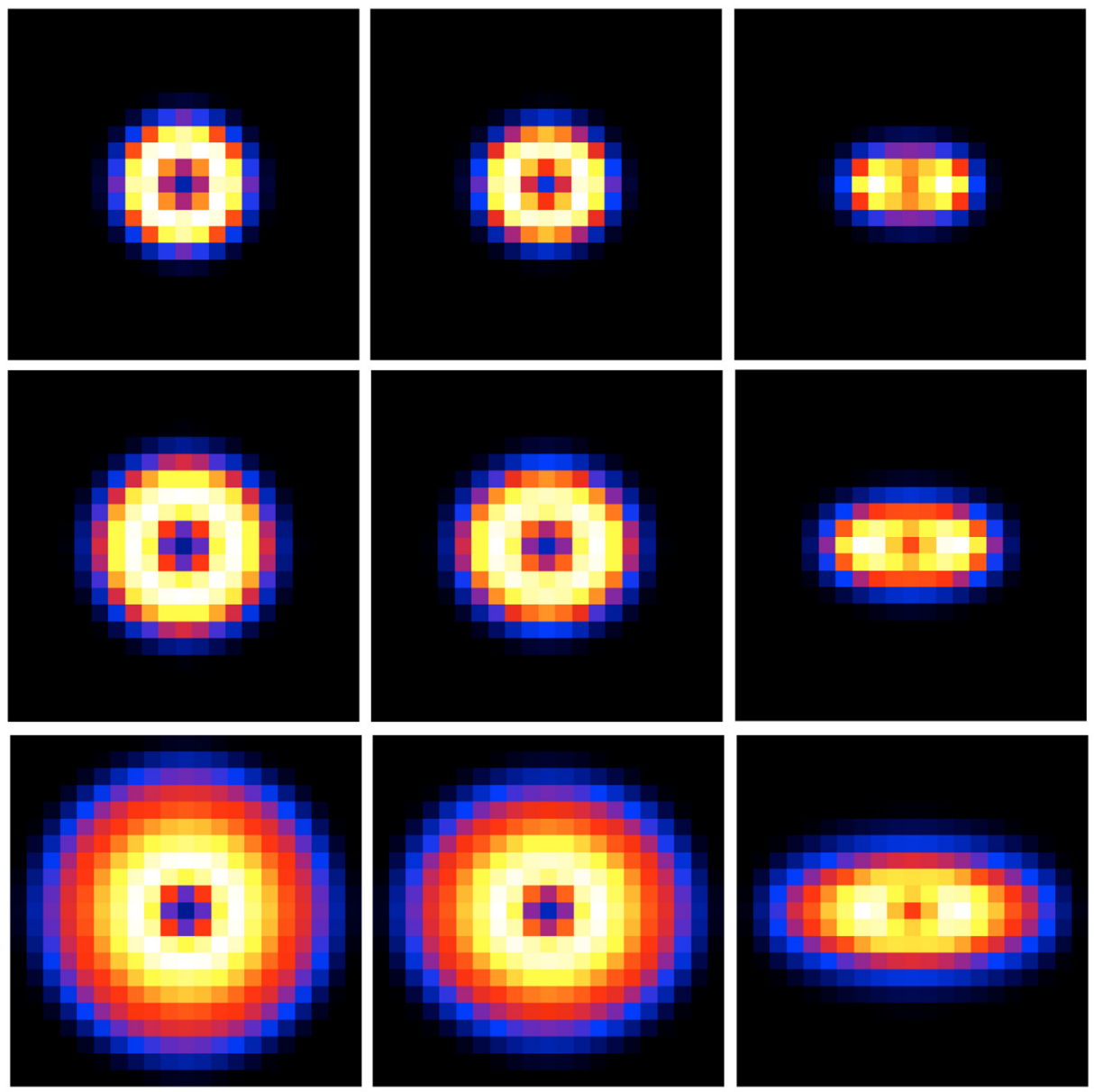

Fig. 3. Model ray tracing images at a wavelength of $350 \mu \mathrm{m}$ generated with the MCFOST code for combinations of three outer disk sizes and disk inclinations. (top row, from left to right) $200 \mathrm{AU}$ outer disk with inclinations of $0^{\circ}, 30^{\circ}$, and $60^{\circ}$. (middle row) $300 \mathrm{AU}$ outer disk with same sequence of inclinations. (bottom row) 500 AU outer disk with same sequence of inclinations. Each image has a size of $1200 \mathrm{AU} \times 1200 \mathrm{AU}$ and the pixels match the size of the CSO map pixels. The dynamic range is a factor of 10 .
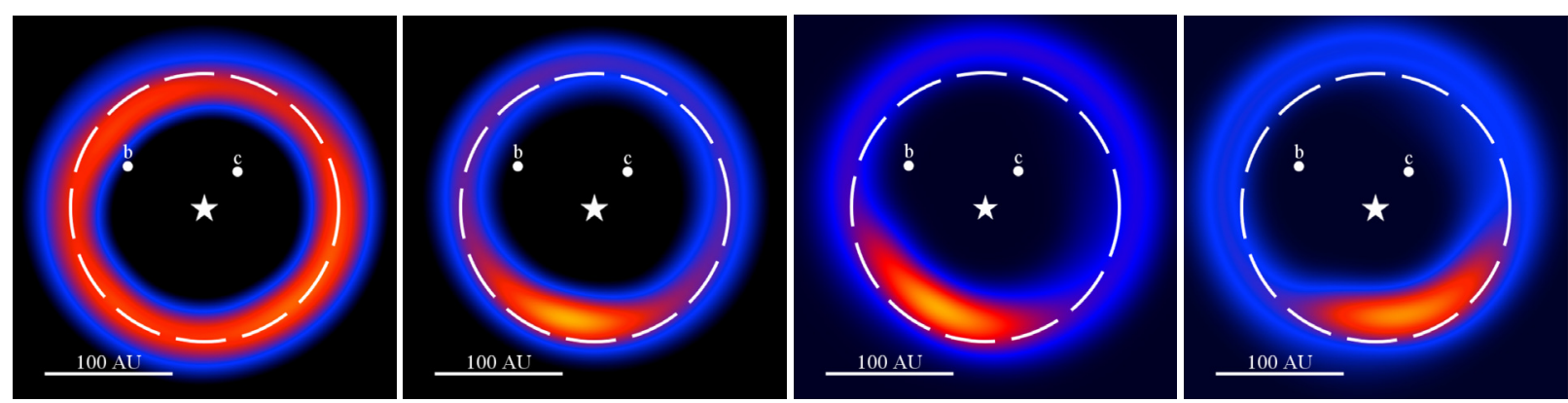

Fig. 4. Simulations of suface density from the $N$-body numerical model at four wavelengths, increasing from left to right: $24 \mu \mathrm{m}, 70 \mu \mathrm{m}, 350 \mu \mathrm{m}$, and $850 \mu \mathrm{m}$. The dynamic range is a factor of 10 , and the simulation is smoothed to a resolution of $11^{\prime \prime} 5$, matching the capability of ALMA. All figures are contemporaneous in the evolution. 